\title{
Natural Convection Flow and Heat Transfer Enhancement of a Nanofluid past a Truncated Cone with Magnetic Field Effect
}

\author{
Sameh E. Ahmed, A. Mahdy \\ Mathematics Department, Faculty of Science, South Valley University, Qena, Egypt \\ Email: sameh_sci_math@yahoo.com,mahdy4@yahoo.com
}

Received August 2, 2012; revised September 1, 2012; accepted September 12, 2012

\begin{abstract}
A nonsimilarity analysis is performed to investigate the laminar, free convection boundary layer flow over a permeable isothermal truncated cone in the presence of a transverse magnetic field effect. A suitable set of dimensionless variables is used and non-similar equations governing the problem are obtained. Fourth order Runge-Kutta with shooting technique is employed for the numerical solution of the obtained equations. Different water-based nanofluids containing $\mathrm{Cu}$, $\mathrm{Ag}, \mathrm{CuO}, \mathrm{Al}_{2} \mathrm{O}_{3}$, and $\mathrm{TiO}_{2}$ are taken into consideration. The effects of pertinent parameters such as the solid volume fraction of nanoparticles, and magnetic field parameter have been investigated. Furthermore, different models of nanofluid based on different formulas for thermal conductivity and dynamic viscosity on the flow and heat transfer characteristics are discussed. Various comparisons with previously published work for the case of a vertical plate are performed and the results are found to be in excellent agreement.
\end{abstract}

Keywords: Nanofluid; Truncated Cone; Magnetic Field; Natural Convection; Non-Similarity Solution

\section{Introduction}

As it is well known that the natural convection phenomena arise in nature as well as in industries. Therefore, laminar free-convection boundary-layer flow of an electrically conducting fluid in the presence of a magnetic field has been investigated by many researchers due to its frequent encounter in industrial and technological applications. For instance, Lin and Chen [1] have studied mixed convection on a vertical plate for fluids on any Prandtl number. The laminar natural convection over a slender vertical frustum of a cone has been reported by $\mathrm{Na}$ and Chiou [2,3]. Chamkha [4] investigated the laminar, coupled heat and mass transfer by natural convective boundary layer flow over a permeable isothermal truncated cone in the presence of magnetic field Raptis and Singh [5] have solved the problem of hydromagnetic natural convection flow past an accelerated vertical plate. Kao [6] has reported on the local nonsimilarity solution for laminar natural convection adjacent to a vertical surface. Na [7] has considered natural convective flow past a nonisothermal vertical flat plate and reported a numerical solution. The laminar natural convection from a nonisothermal cone was analyzed by Roy [8] and Hering and Grosh [9]. An approximate method of solution for the overall heat transfer from vertical cones in laminar natural convection was reported by Alamgir [10]. Takhar and Ram [11] have studied magneto-hydrodynamic natural convection flow of water through a porous medium.

On the other hand, nanofluids have been widely used in industry, because of the growing use of these smart fluids. Many studies [12-17] explained that nanofluids clearly exhibit enhanced thermal conductivity, which goes up with increasing volumetric fraction of nanoparticles. Nanofluid concept is utilized to describe a fluid in which nanometer-sized particles are suspended in conventional heat transfer basic fluids. Conventional heat transfer fluids, including oil, water, and ethylene glycol mixture are poor heat transfer fluids, since the thermal conductivity of these fluids play an important role on the heat transfer coefficient between the heat transfer medium and the heat transfer surface. Therefore, numerous methods have been taken to improve the thermal conductivity of these fluids by suspending nano/micro or larger-sized particle materials in liquids [18]. Choi et al. [19] showed that the addition of a small amount (less than 1\% by volume) of nanoparticles to conventional heat transfer liquids increased the thermal conductivity of the fluid up to approximately two times. Mahdy and Sameh [20] reported numerical analysis for laminar free convection over a vertical wavy surface embedded in a porous medium saturated with a nanofluid. Khan and Pop [21] in- 
vestigated numerically the problem of laminar fluid flow resulting from the stretching of a flat surface in a nanofluid. The used model for the nanofluid incorporated the effects of Brownian motion and thermophoresis. Hojjat et al. [22] investigated experimentally laminar convection heat transfer behavior of three different types of nanofluids flowing through a uniformly heated horizontal circular tube. Nanofluids were made by dispersion of $\mathrm{Al}_{2} \mathrm{O}_{3}, \mathrm{CuO}$, and $\mathrm{TiO}_{2}$ nanoparticles in an aqueous solution of carboxymethyl cellulose (CMC). All nanofluids as well as the base fluid exhibited shear-thinning behaveior.

The goal of the present investigation is to find numerical solutions for the problem of boundary layer flow and heat transfer characteristics utilizing nanofluids past a truncated cone in the presence of a transverse magnetic field effect. The effects due to uncertainties of thermal conductivity and dynamic viscosity have been undertaken and discussed.

\section{Analysis of the Problem}

We consider steady state, laminar, and heat transfer by natural convection, boundary layer flow of an electrically conducting and optically dense fluid about a truncated permeable cone with a half angle $A$ as shown in Figure 1. The fluid is a water based nanofluid containing different types of nanoparticles such as Copper $\mathrm{Cu}$, Silver Ag, Alumina $\mathrm{Al}_{2} \mathrm{O}_{3}$, Copper oxide $\mathrm{CuO}$ and Titanate $\mathrm{TiO}_{2}$. It is assumed that the base fluid and the nanoparticles are in thermal equilibrium and no slip occurs between them. The thermo physical properties of the nanofluid are given in Table $\mathbf{1}$ as $[23,24]$. In addition, the origin of the coordinate system is placed at the vertex of the full cone where $x$ represents the distance along the

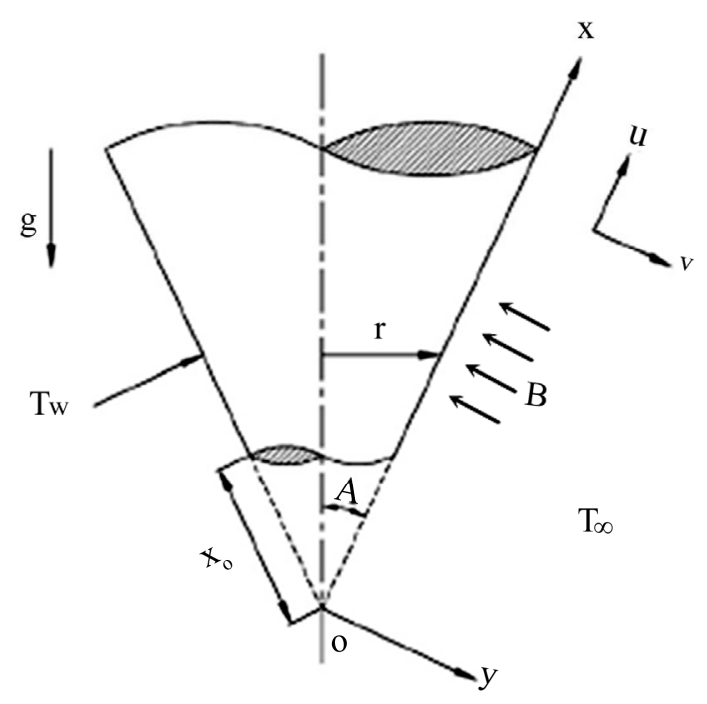

Figure 1. Physical model and coordinate system.
Table 1. Thermo-physical properties of water and nanoparticles $[23,24]$.

\begin{tabular}{ccccc}
\hline & $r_{\left(\mathrm{kgm}^{-3}\right)}$ & $c_{p\left(\mathrm{Jgg}^{-1} \mathrm{~K}^{-1}\right)}$ & $k_{\left(\mathrm{Wm}^{-1} \mathrm{~K}^{-1}\right)}$ & $b_{\mathrm{x} 10^{5}\left(\mathrm{~K}^{-1}\right)}$ \\
\hline $\mathrm{H} 2 \mathrm{O}$ & 997.1 & 4179 & 0.6130 & 21.0 \\
$\mathrm{Cu}$ & 8933 & 385.0 & 401.00 & 1.67 \\
$\mathrm{CuO}$ & 3620 & 531.8 & 76.500 & 1.80 \\
$\mathrm{Ag}$ & 10500 & 235.0 & 429.00 & 1.89 \\
$\mathrm{Al}_{2} \mathrm{O}_{3}$ & 3970 & 765.0 & 40.000 & 0.85 \\
$\mathrm{TiO}_{2}$ & 4250 & 686.2 & 8.9538 & 0.90 \\
\hline
\end{tabular}

cone and $y$ represents the distance normal to the surface of the cone. The cone surface is maintained at a constant temperature $T_{w}$ while the ambient temperature far away from the surface of the cone $T_{\infty}$ is assumed to be uniform. A uniform magnetic field is applied in the $y$-direction normal to the flow direction. The magnetic Reynolds number is assumed to be small so that the induced magnetic field is neglected. In addition, the Hall effect and the electric field are assumed negligible. The small magnetic Reynolds number assumption uncouples the Navier Stokes equations from Maxwell's equations. By invoking all of the boundary layer and Boussineq, the governing equations for this investigation can be written as

$$
\begin{gathered}
\frac{\partial(r u)}{\partial x}+\frac{\partial(r u)}{\partial y}=0 \\
\rho_{n f}\left(u \frac{\partial u}{\partial x}+v \frac{\partial u}{\partial y}\right)=\mu_{n f} \frac{\partial^{2} u}{\partial y^{2}} \\
+g(\beta \rho)_{n f}\left(T-T_{\infty}\right) \cos A-\sigma B^{2} u \\
\left(\rho c_{p}\right)_{n f}\left(u \frac{\partial T}{\partial x}+v \frac{\partial T}{\partial y}\right)=k_{n f} \frac{\partial^{2} T}{\partial y^{2}}
\end{gathered}
$$

The proper boundary and ambient conditions for this problem can be written as

$$
\begin{aligned}
& u=v=0, \quad T=T_{w}, \quad \text { at } \quad y=0 \\
& u=0, \quad T \rightarrow T_{\infty}, \quad \text { at } \quad y \rightarrow \infty
\end{aligned}
$$

where, $u$ and $v$ are the velocity components along the axes $x$ and $y$, respectively, $r$ is the radius of the truncated cone, $\sigma, B$ are the fluid electrical conductivity, magnetic induction. $\rho_{n f}$ is the effective density of the nanofluid, $\mu_{n f}$ is the effective dynamic viscosity of the nanofluid, $T$ is the temperature of the nanofluid, $\beta_{n f}$ is the thermal expansion of the nanofluid, $g$ is the acceleration due to gravity. Now, for nanofluids, let us introducing the expression for $\rho_{n f},\left(\rho c_{p}\right)_{n f}$ and $(\rho \beta)_{n f}$ of the nanofluid as 


$$
\begin{aligned}
& \rho_{n f}=(1-\phi) \rho_{f}+\phi \rho_{s}, \\
& \left(\rho c_{p}\right)_{n f}=(1-\phi)\left(\rho c_{p}\right)_{f}+\phi\left(\rho c_{p}\right)_{s}, \\
& (\rho \beta)_{n f}=(1-\phi)(\rho \beta)_{f}+\phi(\rho \beta)_{s}
\end{aligned}
$$

On the other hand, effective thermal conductivity can be incorporated from the following expression:

$$
k_{n f}=k_{f}\left\{\frac{k_{s}+(n-1) k_{f}-(n-1) \phi\left(k_{f}-k_{s}\right)}{k_{s}+(n-1) k_{f}+\phi\left(k_{f}-k_{s}\right)}\right\}
$$

where $n$ is the empirical shape factor for the nanoparticle. In particular, $n=3$ for spherical shaped nanoparticles and $n=3 / 2$ for cylindrical ones, Table 2 shows four models of nanofluid based on different formulas for thermal conductivity and dynamic viscosity. Furthermore, $\phi$ is the solid volume fraction, $\mu_{f}$ is the dynamic viscosity of the base fluid, $\beta_{f}$ and $\beta_{s}$ are the thermal expansion coefficients of the base fluid and nanoparticle, respectively, $\rho_{f}$ and $\rho_{s}$ are the densities of the base fluid and nanoparticle, respectively, $k_{n f}$ is the thermal conductivity and $\left(\rho c_{p}\right)_{n f}$ is the heat capacity of the nanofluid, $k_{f}$ and $k_{s}$ are the thermal conductivities of the base fluid and nanoparticle, respectively. The governing equations and boundary conditions can be made dimensionless by introducing the stream function such that

$$
r u=\frac{\partial \psi}{\partial y}, \quad r v=-\frac{\partial \psi}{\partial x}
$$

and using the following dimensionless variables

$$
\begin{aligned}
& \xi=\frac{\bar{x}}{x_{0}}=\frac{x-x_{0}}{x_{0}}, \quad \eta=\frac{y}{\bar{x}} G r^{1 / 4}, \\
& \theta(\xi, \eta)=\frac{T-T_{\infty}}{T_{w}-T_{\infty}}, \quad f(\xi, \eta)=\frac{\psi}{r v_{f} G r^{1 / 4}}
\end{aligned}
$$

$$
\begin{aligned}
& u=\frac{v_{f} G r^{1 / 2}}{\bar{x}} f^{\prime}=U_{r} f^{\prime}, \\
& v=-\frac{v_{f} G r^{1 / 4}}{\bar{x}}\left\{\left(\frac{\xi}{1+\xi}+\frac{3}{4}\right) f+\xi \frac{\partial f}{\partial \xi}-\frac{1}{4} \eta f^{\prime}\right\}
\end{aligned}
$$

Substituting Equations (6) and (7) into Equations (2) and (3) yields the following nonsimilar dimensionless equations:

$$
\begin{aligned}
& \frac{1}{(1-\phi)+\phi \frac{\rho_{s}}{\rho_{f}}}\left\{\frac{\mu_{n f}}{\mu_{f}} f^{\prime \prime \prime}-M_{g}^{2} f^{\prime}+\left((1-\phi)+\phi \frac{(\rho \beta)_{s}}{(\rho \beta)_{f}}\right) \theta\right\} \\
& -\frac{1}{2} f^{\prime 2}+\left(\frac{\xi}{1+\xi}+\frac{3}{4}\right) f f^{\prime \prime}=\xi\left(f^{\prime} \frac{\partial f^{\prime}}{\partial \xi}-f^{\prime \prime} \frac{\partial f}{\partial \xi}\right) \\
& \operatorname{Pr}\left\{(1-\phi)+\phi \frac{\left(k_{n f} / k_{f}\right)}{\left(\rho c_{p}\right)_{s}}\right\} \theta^{\prime \prime}+\left(\frac{\xi}{1+\xi}+\frac{3}{4}\right) f \theta^{\prime} \\
& =\xi\left(f^{\prime} \frac{\partial \theta}{\partial \xi}-\theta^{\prime} \frac{\partial f}{\partial \xi}\right) \\
& \eta=0: \quad f(\eta)=f^{\prime}(\eta)=0, \quad \theta(\eta)=1 \\
& \eta \rightarrow \infty: \quad f^{\prime}(\eta) \rightarrow 0, \quad \theta(\eta) \rightarrow 0
\end{aligned}
$$

\begin{tabular}{|c|c|c|c|}
\hline Model & Shape of nanoparticles & Thermal conductivity & Dynamic viscosity \\
\hline I & Spherical & $k_{n f}=k_{f}\left\{\frac{k_{s}+2 k_{f}-2 \phi\left(k_{f}-k_{s}\right)}{k_{s}+2 k_{f}+\phi\left(k_{f}-k_{s}\right)}\right\}$ & $\mu_{n f}=\mu_{f}(1-\phi)^{-2.5}$ \\
\hline II & Spherical & $k_{n f}=k_{f}\left\{\frac{k_{s}+2 k_{f}-2 \phi\left(k_{f}-k_{s}\right)}{k_{s}+2 k_{f}+\phi\left(k_{f}-k_{s}\right)}\right\}$ & $\mu_{\mathrm{nf}}=\mu_{f}\left(1+7.3 \phi+123 \phi^{2}\right)$ \\
\hline III & Cylindrical (nanotubes) & $k_{n f}=k_{f}\left\{\frac{k_{s}+0.5 k_{f}-0.5 \phi\left(k_{f}-k_{s}\right)}{k_{s}+0.5 k_{f}+\phi\left(k_{f}-k_{s}\right)}\right\}$ & $\mu_{n f}=\mu_{f}(1-\phi)^{-2.5}$ \\
\hline IV & Cylindrical (nanotubes) & $k_{n f}=k_{f}\left\{\frac{k_{s}+0.5 k_{f}-0.5 \phi\left(k_{f}-k_{s}\right)}{k_{s}+0.5 k_{f}+\phi\left(k_{f}-k_{s}\right)}\right\}$ & $\mu_{n f}=\mu_{f}\left(1+7.3 \phi+123 \phi^{2}\right)$ \\
\hline
\end{tabular}

where a prime denotes partial differentiation with respect to $\eta$ and

$$
G r=\frac{g \beta_{f}\left(T_{w}-T_{\infty}\right) x^{3}}{v_{f}^{2}}, \operatorname{Pr}=\frac{v_{f}}{\alpha_{f}}, M_{g}^{2}=\frac{\sigma B^{2}}{\mu_{f}} \sqrt{\frac{x_{0}^{4}}{G r_{x}}}
$$

are the Grashof number, Prandtl number and square of the Hartmann number.

Table 2. Models of nanofluid based on different formulas for thermal conductivity and dynamic viscosity. 
The quantities of physical interest are the local skin friction coefficient $C_{f}$ and the rate of heat transfer that expressed in terms of local Nusselt number $N u_{x}$ and these are given by

$$
C_{f}=\frac{2 \tau_{w}}{\rho_{f} U_{r}^{2}}, \quad N u_{x}=\frac{\bar{x} q_{w}}{k_{f}\left(T_{w}-T_{\infty}\right)}
$$

where the skin friction $\tau_{w}$ and the heat transfer from the sheet $q_{w}$ are given by

$$
\tau_{w}=\mu_{n f}\left(\frac{\partial u}{\partial y}\right)_{y=0}, \quad q_{w}=-k_{n f}\left(\frac{\partial T}{\partial y}\right)_{y=0}
$$

Applying the non-dimensional transformations (5), one obtain

$$
\begin{aligned}
& C_{f} G r_{x}^{\frac{1}{4}}=2\left(\frac{\mu_{n f}}{\mu_{f}}\right) f^{\prime \prime}(\xi, 0), \\
& N u_{x} G r_{x}^{-\frac{1}{4}}=-\left(\frac{k_{n f}}{k_{f}}\right) \theta^{\prime}(\xi, 0)
\end{aligned}
$$

\section{Numerical Procedure}

The numerical algorithm used to solve the dimensionless equations (8) and (9) with the boundary conditions (10) is based on the well-known fourth order Runge-Kutta integration scheme. In the current technique, the difference between the forward and backward values of dependent variables should be zero for a true solution. The procedure uses a generalized Newton method to reduce these differences to zero, by calculating corrections to the estimated boundary values. This process is repeated iteratively until convergence is obtained to as specified accuracy. This method was found to be suitable and gave results that are very close to the results obtained in Refs.
$[4,25,26]$. Table 3 shows comparison of values of $f^{\prime \prime}(0,0)$ and $\theta^{\prime}(0,0)$ for various values of Pr for pure fluid. As it is observed, there is an excellent agreement with the earlier published results.

\section{Results and Discussion}

Steady state two-dimensional laminar magneto-hydrodynamics natural convection flow and heat transfer of a nanofluid past a truncated cone has been studied numerically. Parametric studies of the influence of various parameters such as finite volume fraction $(0 \leq \phi \leq 0.15)$, magnetic field parameter $\left(0 \leq M_{g} \leq 5\right)$, different formulas of dynamic viscosity ratio $\left(\mu_{n f} / \mu_{f}\right)$ and thermal conductivity ratio $\left(k_{n f} / k_{f}\right)$ on the fluid flow and heat transfer have been performed. In all the obtained results, pure water with $\operatorname{Pr}=7$ has been used as a base nanofluid. Figures 2 and $\mathbf{3}$ display the effects of solid volume fraction on the local skin friction coefficient and the rate of heat transfer for Cu-water nanofluid using model I. It is found that, increasing in solid volume fraction results in an increase in rate of heat transfer and a reduction in local skin friction coefficient. In fact, high values of $\phi$ causes the fluid becomes more viscous. As results, the natural convection is reduced which causes the fluid flows is very slowly. This velocity reduction causes an increase in thermal boundary layer thickness, which in turn, increases the rate of heat transfer and decreases the local Nusselt number.

The main objective from adding nanoparticles to the classical fluid is enhancing the rate of heat transfer for such fluids, so it is necessary to explain which the best model can be used to reach to this goal. In Figures $\mathbf{4}$ and 5, a comparison among different formulas for nanofluid

\begin{tabular}{|c|c|c|c|c|c|c|c|c|}
\hline \multirow{2}{*}{$\operatorname{Pr}$} & \multicolumn{4}{|c|}{$f^{\prime \prime}(0,0)$} & \multicolumn{4}{|c|}{$-\theta^{\prime}(0,0)$} \\
\hline & [25] & [26] & [4] & present & [25] & [26] & {$[4]$} & present \\
\hline 0.0001 & 1.4998 & - & 1.4997 & 1.49906 & 0.0060 & - & 0.0059 & 0.00598 \\
\hline 0.001 & 1.4728 & - & 1.4727 & 1.47299 & 0.0189 & - & 0.0188 & 0.01859 \\
\hline 0.01 & 1.3968 & - & 1.3965 & 1.38941 & 0.0570 & - & 0.0574 & 0.05738 \\
\hline 0.1 & 1.2144 & 1.2104 & 1.2151 & 1.20918 & 0.1629 & 0.1637 & 0.1630 & 0.16312 \\
\hline 1.0 & 0.9084 & 0.9081 & 0.9081 & 0.90813 & 0.4012 & 0.4009 & 0.4015 & 0.40131 \\
\hline 10.0 & 0.5927 & 0.5930 & 0.5927 & 0.59268 & 0.8266 & 0.8266 & 0.8274 & 0.82664 \\
\hline 100.0 & 0.3539 & 0.3564 & 0.3558 & 0.35557 & 1.5493 & 1.5495 & 1.5503 & 1.54928 \\
\hline 1000.0 & 0.2049 & - & 0.2049 & 0.20468 & 2.8035 & - & 2.8044 & 2.80099 \\
\hline 10000.0 & 0.1161 & - & 0.1161 & 0.11669 & 5.0127 & - & 5.0131 & 5.02900 \\
\hline
\end{tabular}
dynamic viscosity and nanofluid thermal conductivity was performed at $\phi=0.08$ and $M_{g}=1$. These formulas

Table 3. Comparison of values of $f^{\prime \prime}(0,0)$ and $-\theta^{\prime}(0,0)$ for various values of Pr for pure fluid. 


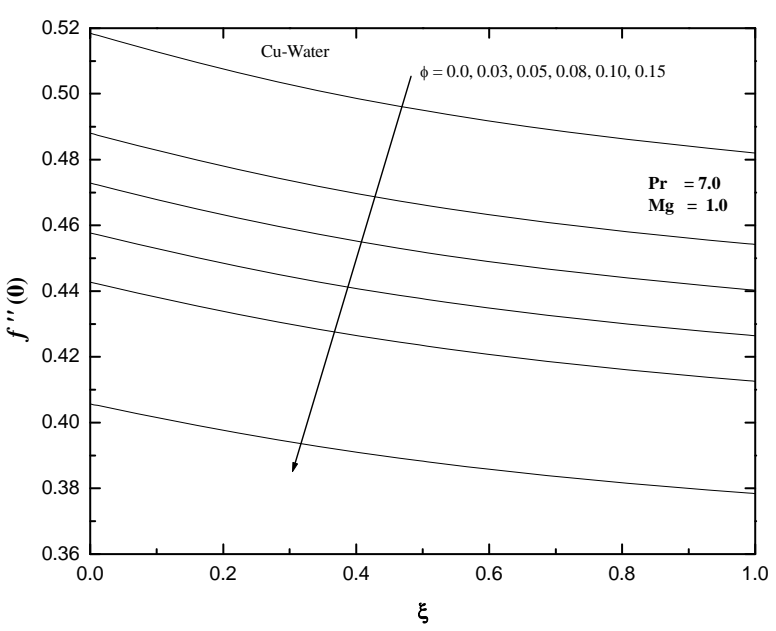

Figure 2. Profiles of skin-friction coefficient for different values of volume fraction.

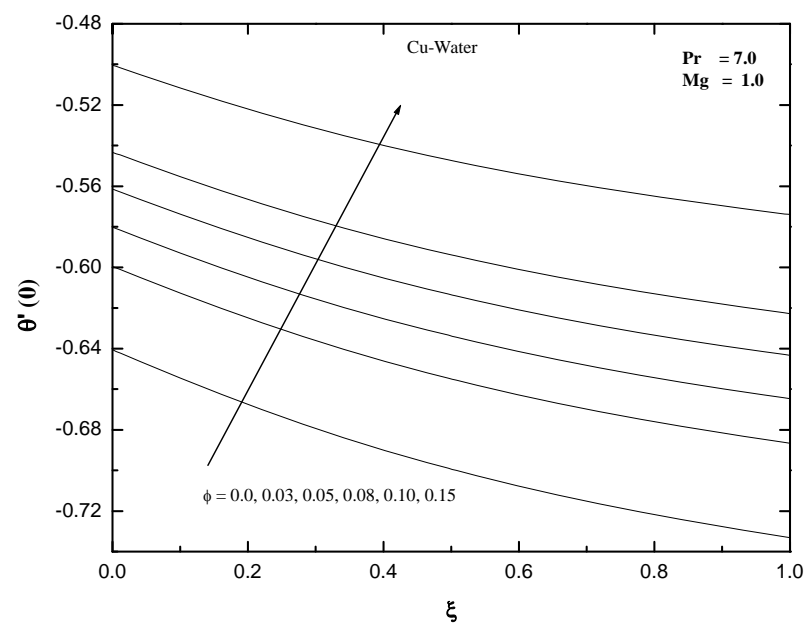

Figure 3. Profiles of local Nusselt number for different values of volume fraction.

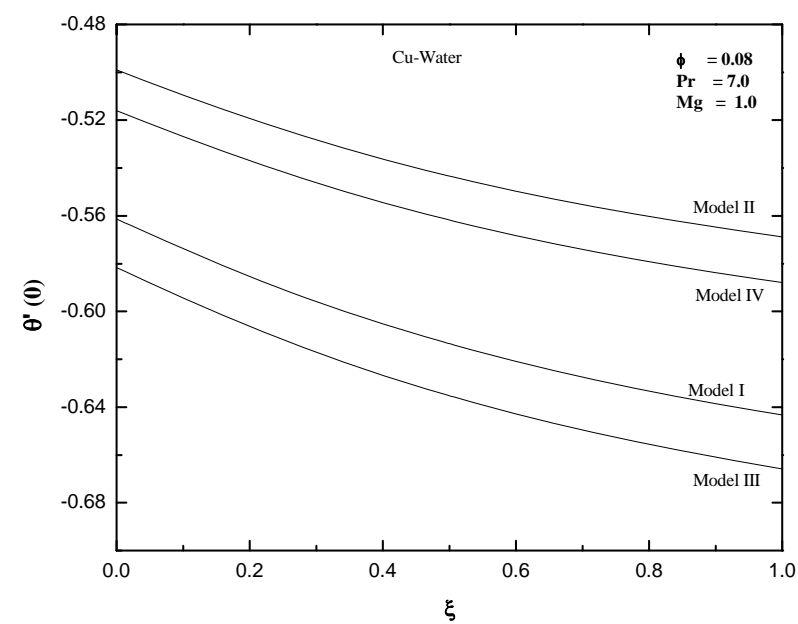

Figure 4. Profiles of local Nusselt number for different models. were represented in the present study by model I, model II, model III and model IV (Table 2). It is clear that, spherical nanoparticles which represented by model II give larger rate of heat transfer than model I. On the contrary, cylindrical nanoparticles represented by model III give lower rate of heat transfer than model IV. Also, spherical and cylindrical shapes of nanoparticles given by model I and model III tend to decrease dynamic viscosity of the nanofluid which increase the local skin friction coefficient compared with model II and model IV.

Figures 6-9 display the effects of magnetic field parameter $\left(0 \leq M_{g} \leq 5\right)$ on velocity profiles, temperature distributions, local skin friction coefficient and rate of heat transfer for pure water and Cu-water nanofluid using model I, respectively. It can be observed that, increasing in magnetic field parameter results in a reduction in the fluid motion and an increase in the fluid temperature.

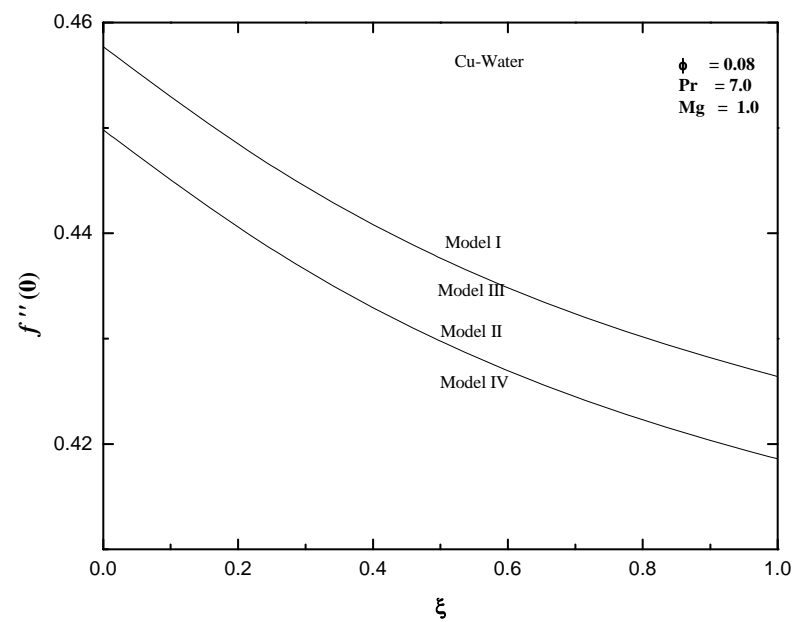

Figure 5. Profiles of skin-friction coefficient for different models.

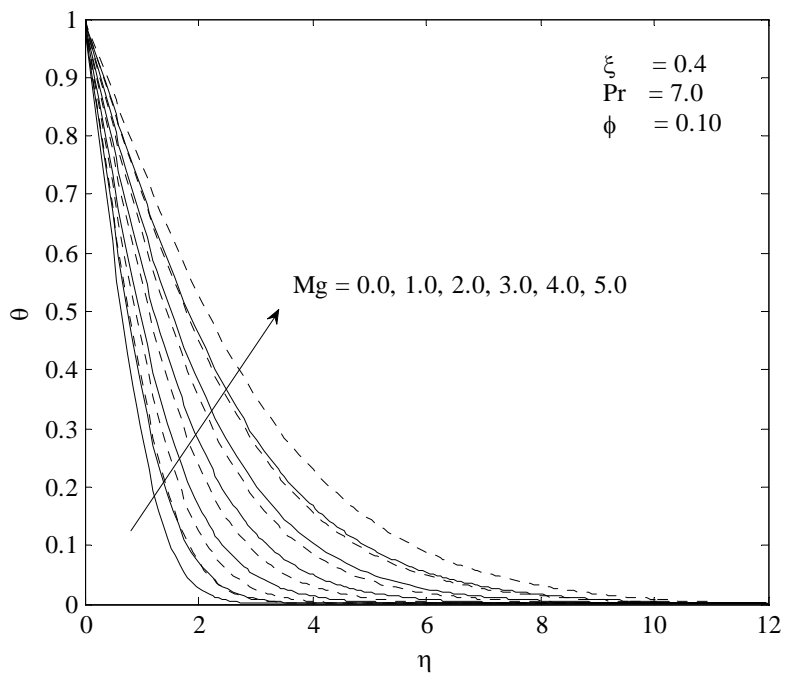

Figure 6. Profiles of fluid temperature for different values magnetic field parameter Mg (-pure water , .. Cu-Water). 


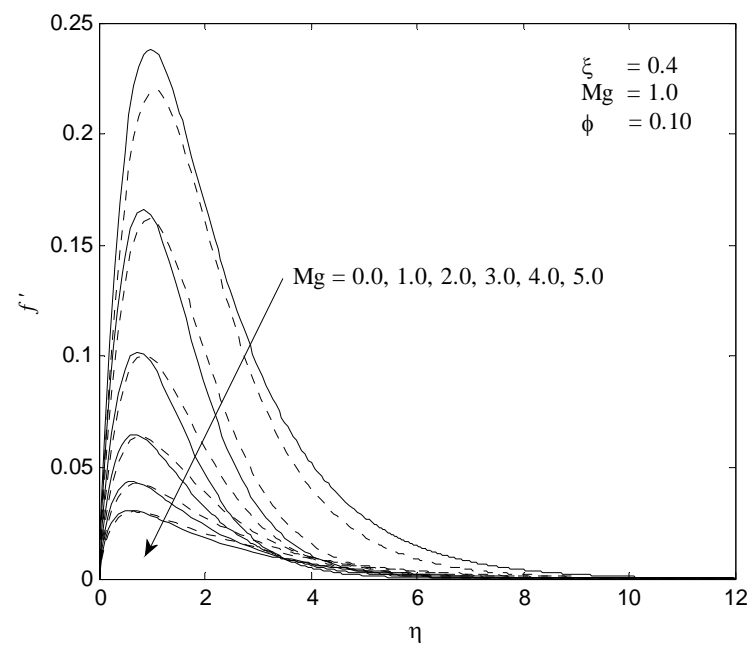

Figure 7. Velocity profiles for different values magnetic field parameter Mg (一pure water, $\cdots$ Cu-Water).

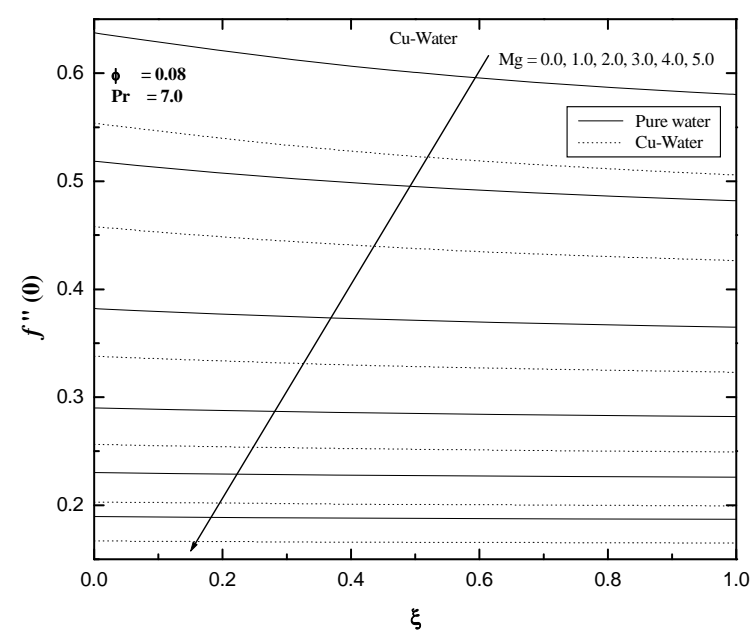

Figure 8. Profiles of skin-friction coefficient for different values of magnetic field $\mathrm{Mg}$.

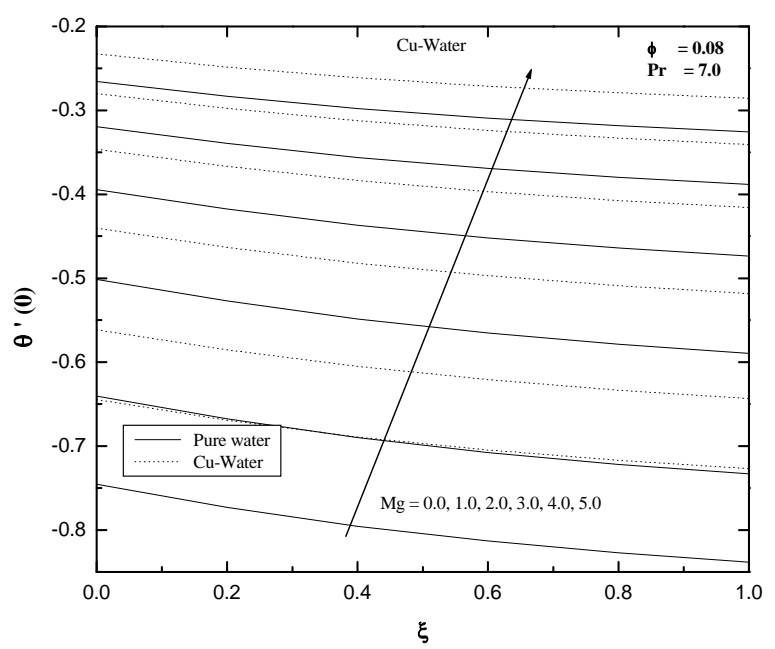

Figure 9. Profiles of local nusselt number for different values of magnetic field $\mathrm{Mg}$.
This can be attributed to the existence of magnetic field within the flow region which causes a force called Lorentz force. This force works opposite to the flow direction and it resists the flow. This reduction leads to decreases the local skin friction coefficient and increases the boundary layer thickness which in turn, increases the rate of heat transfer as well.

Figures $\mathbf{1 0}$ and $\mathbf{1 1}$ show the profiles of fluid velocity and fluid temperature for pure water $(\phi=0)$, Cu-water, $\mathrm{CuO}$-water, $\mathrm{Al}_{2} \mathrm{O}_{3}$-water, Ag-water and $\mathrm{TiO}_{2}$-water nanofluid with $(\phi=0.08)$ related with Model I at $M_{g}=1$ and $\xi=0.4$. The results show that, the fluid motion becomes very slowly by adding $\mathrm{Al}_{2} \mathrm{O}_{3}$ nanoparticles. However, the $\mathrm{CuO}$-nanoparticles give faster motion for nanofluid than other nanoparticles. On the other hand, the high value of thermal conductivity of Ag (Table 1) causes to decrease the fluid temperature whereas, the $\mathrm{TiO}_{2}$ nanoparticles leads to increase the fluid temperature. The behaviors of velocity and temperatures mentioned above have opposite effects on behaviors of local skin friction coefficient and rate of heat transfer. It can be noticed from Figures $\mathbf{1 2}$ and $\mathbf{1 3}$ which depicted the profiles of local skin friction coefficient and rate of heat transfer for different nanoparticles, respectively, that, $\mathrm{TiO}_{2}$-water nanofluid has a high value of skin friction while the $\mathrm{CuO}$-water nanofluid has a lower value of it. In addition, the high rate of heat transfer can be obtained by adding Ag-nanoparticles and $\mathrm{TiO}_{2}$ nanoparticles give a lower value of it. The mean responsible of these effects is the values of thermal conductivity for such nanoparticles (Table 1). Similar behaviors are observed by Rana and Bhargava [27] which make sure that the present results are more accurate.

\section{Conclusion}

In this paper, the problems of MHD natural convection

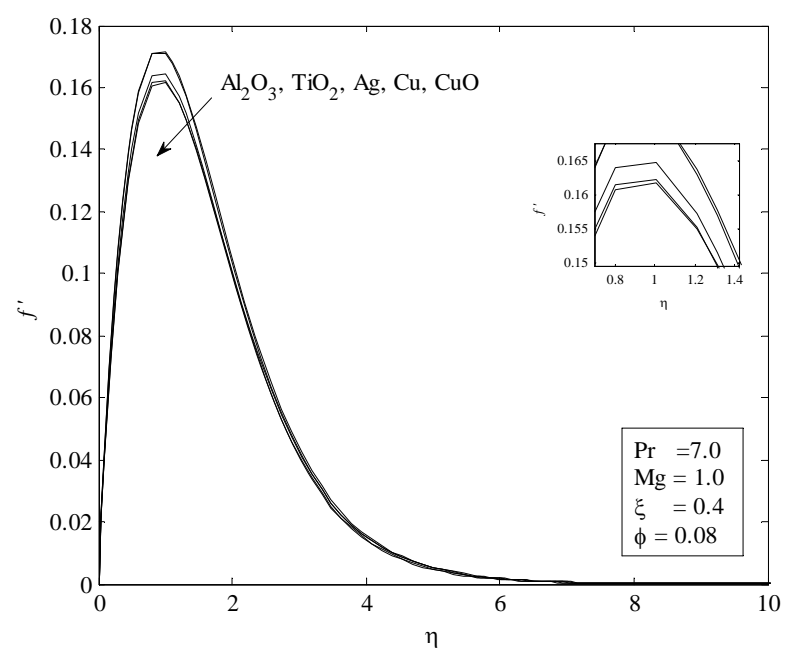

Figure 10. Velocity profiles for different nanoparticles. 


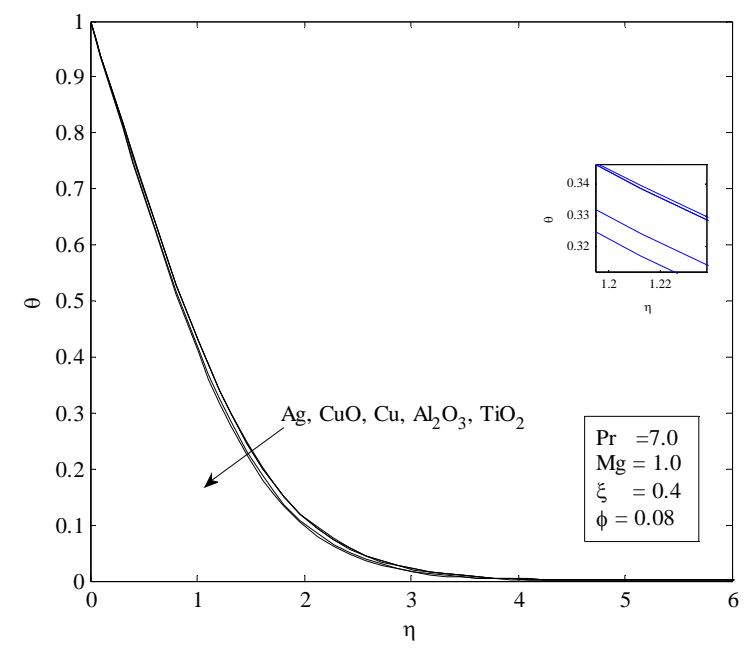

Figure 11. Profiles of fluid temperature for different nanoparticles.

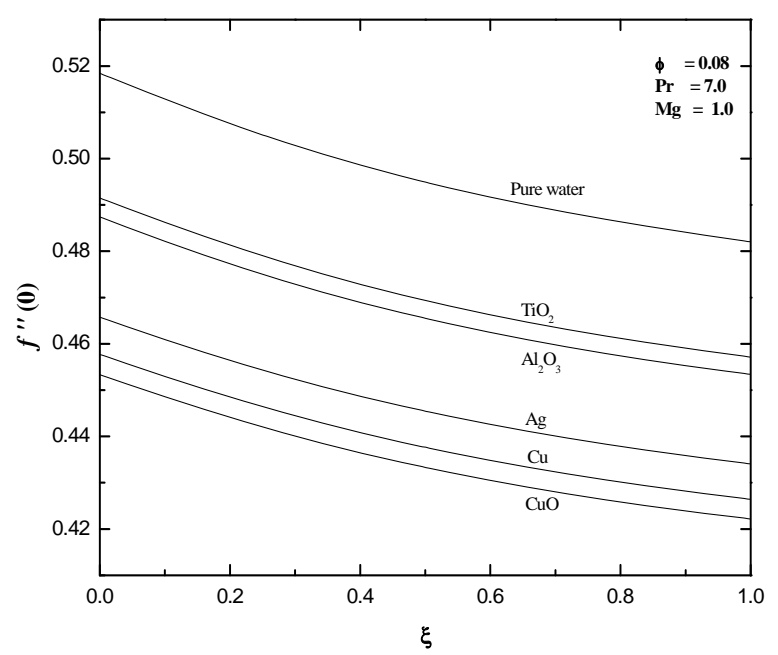

Figure 12. Profiles of skin-friction coefficient for different nanoparticles.

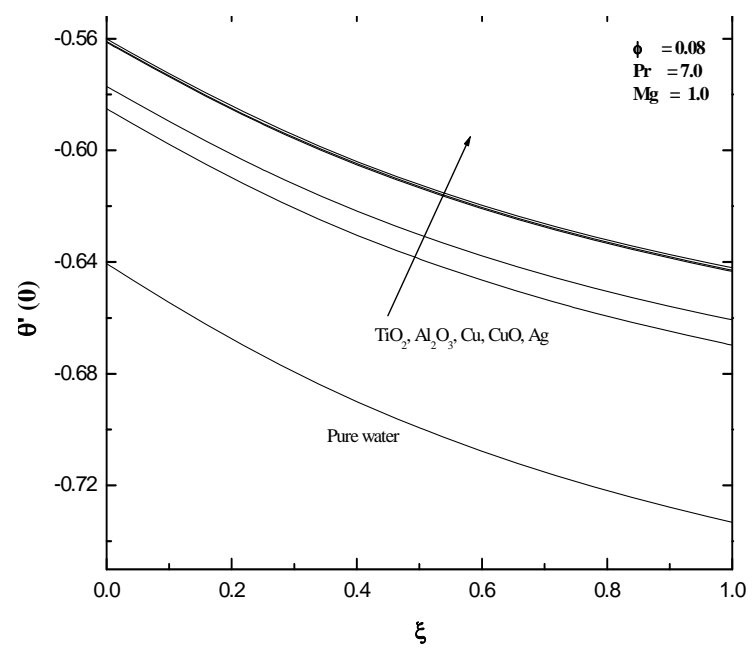

Figure 13. Profiles of local nusselt number for different nanoparticles and heat transfer of a nanofluid past a truncated cone are investigated. The governing partial differential equations for mass, momentum and energy are transformed to non-similar equations by using a non-dimensional transformation. These equations are solved numerically using the well known fourth order Runge-Kutta method. The effects of solid volume fraction, magnetic field parameter, different nanoparticles and different formulas of thermal conductivity and dynamic viscosity are discussed. It is found that, as the solid volume fraction increases, the rate of heat transfer increases whereas the local skin friction coefficient takes the inverse behaviors. Model II (spherecal nanoparticles) is found to be the best model for enhancing the rate of heat transfer compared with other models. In addition, increasing in magnetic field parameter leads to decrease both of the velocity and local skin friction coefficient and increase the fluid temperature as well as the rate of heat transfer. Finally, among all different type of nanoparticles given in this study, Ag-nanoparticles give a higher rate of heat transfer and $\mathrm{TiO}_{2}$ nanoparticles have a lower value of it.

\section{REFERENCES}

[1] H. T. Lin and C. C. Chen, "Mixed Convection on Vertical Plate for Fluids of Any Prandtl Number," Heat and Mass Transfer, Vol. 22, No. 3-4, 1988, pp. 159-168. doi:10.1007/BF01052981

[2] T. Y. Na and J. P. Chiou, "Laminar Natural Convection over a Slender Vertical Frustum of a Cone,” Heat and Mass Transfer, Vol. 12, No. 2, 1979, pp. 83-87. doi:10.1007/BF01002323

[3] T. Y. Na and J. P. Chiou, "Laminar Natural Convection over a Frustum of a Cone," Applied Scientific Research, Vol. 35, No. 5-6, 1979, pp. 409-421. doi:10.1007/BF00420389

[4] A. J. Chamkha, "Coupled Heat and Mass Transfer by Natural Convection about a Truncated Cone in the Presence of Magnetic Field and Radiation Effects,” Numerical Heat Transfer, Part A, Vol. 39, No. 5, 2001, pp. 511-530.

[5] A. Raptis and A. K. Singh, "MHD Free Convection Flow Past an Accelerated Vertical Plate," International Communications in Heat and Mass Transfer, Vol. 10, No. 4, 1983, pp. 313-321. doi:10.1016/0735-1933(83)90016-7

[6] T. T. Kao, "Local Nonsimilar Solution for Laminar Free Convection Adjacent to a Vertical Wall,” Transactions of ASME Journal of Heat Transfer, Vol. 98, No. 2, 1976, pp. 321-322. doi:10.1115/1.3450544

[7] T. Y. Na, "Numerical Solution of Natural Convection Flow Past a Non-Isothermal Vertical Flat Plate,” Applied Scientific Research, Vol. 33, No. 5-6, 1978, pp. 519-543.

[8] S. Roy, "Free Convection from a Vertical Cone at High Prandtl Numbers," Transactions of ASME Journal of Heat Transfer, Vol. 96, No. 1, 1974, pp. 115-117. doi:10.1115/1.3450128

[9] R. G. Hering and R. J. Grosh, "Laminar Free Convection 
from a Non-Isothermal Cone at Low Prandtl Number,” International Journal of Heat and Mass Transfer, Vol. 8, No. 10, 1965, pp. 1333-1337. doi:10.1016/0017-9310(65)90059-1

[10] M. Alamgir, “Over-All Heat Transfer from Vertical Cones in Laminar Free Convection: An Approximate Method," Transactions of ASME Journal of Heat Transfer, Vol. 101, No. 1, 1979, pp. 174-176. doi:10.1115/1.3450912

[11] H. S. Takhar and P. C. Ram, "Magnetohydrodynamics Free Convection Flow of Water at $4^{\circ} \mathrm{C}$, through a Porous Medium," International Communications in Heat and Mass Transfer, Vol. 21, No. 3, 1994, pp. 371-376. doi:10.1016/0735-1933(94)90005-1

[12] Y. Xuan and O. Li, "Heat Transfer Enhancement of Nanofluids," International Journal of Heat and Fluid Flow, Vol. 21, No. 1, 2000, pp. 58-64. doi:10.1016/S0142-727X(99)00067-3

[13] Q. Li and Y. Xuan, "Experimental Investigation of Transport Properties of Nanofluids,” In: B. X. Wang, Ed., Heat Transfer Science \& Technology, Higher Education Press, Beijing, 2000, pp. 757-784.

[14] D. Wen and Y. Ding, "Experimental Investigation into Convective Heat Transfer of Nanofluids at the Entrance Region under Laminar Flow Conditions, International Journal Heat and Mass Transfer, Vol. 47, No. 24, 2004, pp. 5181-5188. doi:10.1016/j.ijheatmasstransfer.2004.07.012

[15] P. Bhattacharya, S. Saha, A. Yadav, P. Phelan and R. Prasher, "Brownian Dynamics Simulation to Determine the Effect Thermal Conductivity of Nanofluids," Journal of Applied Physics, Vol. 95, No. 11, 2004, pp. 6492-6494. doi:10.1063/1.1736319

[16] A. Mokmeli and M. Saffar-Avval, "Prediction of Nanofluid Convective Heat Transfer Using the Dispersion Model," International Journal of Thermal Sciences, Vol. 49, No. 3, 2010, pp. 471-478. doi:10.1016/j.ijthermalsci.2009.09.005

[17] M. Mansour, R. Mohamed, M. Abd-Elaziz and S. Ahmed, "Numerical Simulation of Mixed Convection Flows in a Square Lid-Driven Cavity Partially Heated from Below Using Nanofluid,” International Communications in Heat and Mass Transfer, Vol. 37, No. 10, 2010, pp. 1504-1512. doi:10.1016/j.icheatmasstransfer.2010.09.004

[18] R. Lotfi, Y. Saboohi and A. Rashidi, "Numerical Study of Forced Convective Heat Transfer of Nanofluids: Com- parison of Different Approaches,” International Communications in Heat and Mass Transfer, Vol. 37, No. 1, 2010, pp. 74-78. doi:10.1016/j.icheatmasstransfer.2009.07.013

[19] S. Choi, Z. Zhang, W. Yu, F. Lockwood and E. Grulke, "Anomalously Thermal Conductivity Enhancement in Nanotube Suspensions,” Applied Physics Letter, Vol. 79, No. 14, 2001, pp. 2252-2254. doi:10.1063/1.1408272

[20] A. Mahdy and S. E .Ahmed, "Laminar Free Convection over a Vertical Wavy Surface Embedded in a Porous Medium Saturated with a Nanofluid," Transport in Porous Media, Vol. 91, No. 2, 2012, pp. 423-435. doi:10.1007/s11242-011-9852-4

[21] W. Khan and I. Pop, "Boundary-Layer Flow of a Nanofluid Past a Stretching Sheet," International Journal of Heat and Mass Transfer, Vol. 53, No. 11-12, 2010, pp. 2477-2483. doi:10.1016/j.ijheatmasstransfer.2010.01.032

[22] M. Hojjat, S. Etemad and R. Bagheri, "Laminar Heat Transfer of Non-Newtonian Nanofluids in a Circular Tube,” Korean Journal of Chemical Engineering, Vol. 27, No. 5, 2010, pp. 1391-1396. doi:10.1007/s11814-010-0250-3

[23] H. F. Oztop and E. Abu-Nada, "Numerical Study of Natural Convection in Partially Heated Rectangular Enclosures Filled with Nanofluids," International Journal of Heat Fluid Flow, Vol. 29, No. 5, 2008, pp. 1326-1336. doi:10.1016/j.ijheatfluidflow.2008.04.009

[24] Z. Alloui, P. Vasseur and M. Reggio, "Natural Convection of Nanofluids in a Shallow Cavity Heated from Below," International Journal of Thermal Sciences, Vol. 50, No. 3, 2011, pp. 385-393. doi:10.1016/j.ijthermalsci.2010.04.006

[25] K. A. Yih, "Effect of Radiation on Natural Convection about a Truncated Cone," International Journal of Heat Mass Transfer, Vol. 42, No. 23, 1999, pp. 4299-4305. doi:10.1016/S0017-9310(99)00092-7

[26] T. Cebeci and P. Bradshaw, "Physical and Computational Aspects of Convective Heat Transfer," Springer, New York, 1984, p. 270.

[27] P. Rana and R. Bhargava, "Numerical Study of Heat Transfer Enhancement in Mixed Convection Flow along a Vertical Plate with Heat Source/Sink Utilizing Nanofluids, Communications in Nonlinear Science and Numerical Simulation, Vol. 16, No. 11, 2011, pp. 4318-4334. doi:10.1016/j.cnsns.2011.03.014 